\title{
Tagungsbericht
}

Monika Linne* und Uwe Jensen

\section{Präsentation und Live-Demo von SowiDataNet - Fachrepositorium für sozial- und wirtschaftswissenschaftliche Forschungsdaten}

DOI 10.1515/iwp-2017-0033

Die Partner des Projektes luden am 10. Februar 2017 zur Vorstellung von SowiDataNet in das DIW nach Berlin ein. SowiDataNet ist ein aus Mitteln des Leibniz-Wettbewerbs gegründeter Projektverbund, zu dem sich GESIS - LeibnizInstitut für Sozialwissenschaften (Projektleitung), WZB Wissenschaftszentrum Berlin für Sozialforschung, DIW Berlin - Deutsches Institut für Wirtschaftsforschung und ZBW - Deutsche Zentralbibliothek für Wirtschaftswissenschaften zusammengeschlossen haben. Die Veranstaltung wurde von Mathis Fräßdorf (WZB Berlin) moderiert, der etwa 70 Gäste aus 44 Einrichtungen begrüßen konnte.

Das Programm wurde mit einem Grußwort von Angelica E. Röhr, Geschäftsführerin des DIW, eröffnet, die zunächst die gute Kooperation der Projektpartner hervorhob. Deren erfolgreiche Entwicklung von SowiDataNet ermögliche es, Forschungsinstituten und universitären Fachbereichen der Sozial- und Wirtschaftswissenschaften ohne eigene Infrastruktur ein System anzubieten, mit dem die dort Forschenden ${ }^{1}$ ihre Forschungsdaten eigenständig und zuverlässig sichern und zitierfähig für die Nachnutzung bereitstellen können.

Anita Eppelin (Referentin für die Themen Open Access und Forschungsdaten in der Leibniz-Gemeinschaft) beleuchtete anschließend den „Mehrwert einer koordiniertdezentralen Forschungsgemeinschaft im Hinblick auf Forschungsdaten in der Leibniz-Gemeinschaft“. Ausgehend

1 Aus Gründen der besseren Lesbarkeit wird im Text, wann immer möglich, eine neutrale Geschlechtsform gewählt. In allen anderen Fällen wird abwechselnd die weibliche und die männliche Form verwendet. Gemeint sind stets alle Geschlechter.

*Kontaktperson: Monika Linne, GESIS - Leibniz-Institut für Sozialwissenschaften, Unter Sachsenhausen 6-8, 50667 Köln, E-Mail:monika.linne@gesis.org, http://orcid.org/0000-0003-3579-6005

Uwe Jensen, GESIS - Leibniz-Institut für Sozialwissenschaften, Unter Sachsenhausen 6-8, 50667 Köln, E-Mail: uwe.jensen@gesis.org, http://orcid.org/0000-0003-3526-6522 von den Herausforderungen einer stetig steigenden „Datenflut“ im Kontext von Digitalem Wandel und Open Science präsentierte sie einen breiten Überblick über die unterschiedlichen Initiativen von forschungsfördernden und Wissenschaftsorganisationen auf nationaler und internationaler Ebene. Um diesen Herausforderungen zu begegnen, hob Eppelin vier Lösungsansätze hervor, die sie anhand von Beispielen illustrierte. Erstens gelte es, Infrastrukturen $\mathrm{zu}$ vernetzen und vertrauenswürdig und nachhaltig zu gestalten, etwa durch Anwendung der FAIR Data Principles ${ }^{2}$ oder des Data Seal of Approval ${ }^{3}$. Weiterhin seien digitale Kompetenzen in der universitären Ausbildung sowie neue Ausbildungsgänge erforderlich, um datenbezogene Qualifizierungen zu stärken. Drittens sollten rechtliche Rahmenbedingungen dem wissenschaftlichen Bedarf angepasst werden, um Data Sharing-Aktivitäten zu fördern. Schließlich sei eine Anerkennungskultur zu entwickeln, welche die vorherrschenden Faktoren für den Austausch von Forschungsdaten berücksichtigt, wie sie etwa von Fecher, B./Friesike, S./Hebing, M. (2015) ${ }^{4}$ untersucht wurden.

Den Mehrwert einer koordiniert-dezentralen Forschungsgemeinschaft betrachtet Eppelin abschließend im Kontext der Leibniz-Roadmap „Forschungsinfrastrukturen“. Sie spiele eine zentrale Rolle, indem die Potentiale und Stärken von Leibniz-Partnern aus unterschiedlichen Disziplinen in gemeinsamen Projektkonzepten gebündelt würden. So unterstreiche auch das Leibniz Network for Open Research Data (LEIBNIZ DATA) ${ }^{5}$ den Anspruch der Leibniz-Gemeinschaft, „Kümmerer“ für Forschungsdaten nach innen und nach außen zu sein.

2 https://www.force11.org/group/fairgroup/fairprinciples.

$3 \mathrm{http://www.datasealofapproval.org/en/.}$

4 Fecher, B./Friesike, S./Hebing, M. (2015) What Drives Academic Data Sharing? PLoS ONE 10(2): e0118053. doi:10.1371/journal.pone.0118053.

5 https://www.leibniz-gemeinschaft.de/infrastrukturen/leibniz-road map-forschungsinfrastrukturen/leibniz-data/. 
Deshalb wird auch das Projekt SowiDataNet in der Förderlinie 2: „Nationale und internationale Vernetzung“ des Leibniz-Wettbewerbs 2014 gefördert. Eines der übergeordneten Projektziele ist „die Integration dezentral erzeugter Forschungsdaten unter Nutzung einer gemeinsamen Infrastruktur. Dies hat den Vorteil, dass Forschungsdaten der Sozial- und Wirtschaftswissenschaften in Deutschland nach einheitlichen Standards archiviert und dokumentiert werden, zitierbar und zugänglich gemacht werden können.“ (Droß, P./Linne M.2016: 651) ${ }^{6}$

Kerstin Helbig (Koordinatorin Forschungsdatenmanagement HU Berlin) berichtete anhand der Erfahrungen der Humboldt-Universität zu Berlin über „Forschungsdatenmanagement in der Praxis“. Seit 2012 wird dort durch interne Kooperationen, gestützt auf einer empirischen Erhebung (Simukovic et. al. 2013) unter den Forschenden, die Basis eines universitären Forschungsdatenmanagements geschaffen. Um den Anforderungen „Guter Wissenschaftlicher Praxis“ gerecht zu werden, wurden Grundsätze zum Umgang mit Forschungsdaten und unterstützende Handlungsempfehlungen entwickelt. Für die Forschenden seien viele praktische Angebote entwickelt worden, die sich von der Beratung zum Thema Forschungsdaten über die Vermittlung von technischen Diensten bis hin zur Unterstützung bei der Aufstellung von Datenmanagementplänen erstrecken. Seit 2015 werden zielgruppenorientierte Workshops zum Datenmanagement angeboten und durch umfangreiche Informationen im Web ${ }^{8}$ ergänzt.

Als Fazit der bisherigen Aktivitäten stellte Helbig u.a. heraus, dass die heterogene Datenlandschaft einen fachspezifischen Umgang mit den Forschungsdaten erfordere. Dabei stehe der Bedarf an praktischer Unterstützung, z.B. durch die Bereitstellung einfacher Werkzeuge zur Datenund Mediensicherung, im Vordergrund. Gleichzeitig steige die Nachfrage nach Beratungen zum Thema von Datenmanagementplänen. Studierende und Promovierende seien insgesamt offener für das Thema Forschungsdatenmanagement. Deren Bedarf greife das DFG-geförderte Projekt „Elektronische Dissertationen Plus (eDissPlus)“ auf, das u. a. „konzeptionelle und technische Anforderungen für eine zeitgemäße Archivierung und Publikation von

6 Patrick J. Droß, Monika Linne. Sicheres und einfaches Data Sharing mit SowiDataNet: dokumentieren - veröffentlichen - nachnutzen. Seite 651. In: Bibliotheksdienst. Band 50, Heft 7, Seiten 649-660. Juni 2016. DOI 10.1515/bd-2016-0079.

7 Simukovic, E./Kindling, M./Schirmbacher, P. (2013): Ergebnisse der Umfrage zum Umgang mit digitalen Forschungsdaten an der Humboldt-Universität zu Berlin. Zenodo. https://doi.org/10.5281/ze nodo.7446.

8 https://www.cms.hu-berlin.de/de/dl/dataman.
Forschungsdaten entwickelt, die im Rahmen von Dissertationen entstehen“ (https://www2.hu-berlin.de/edissplus/: o.S.).

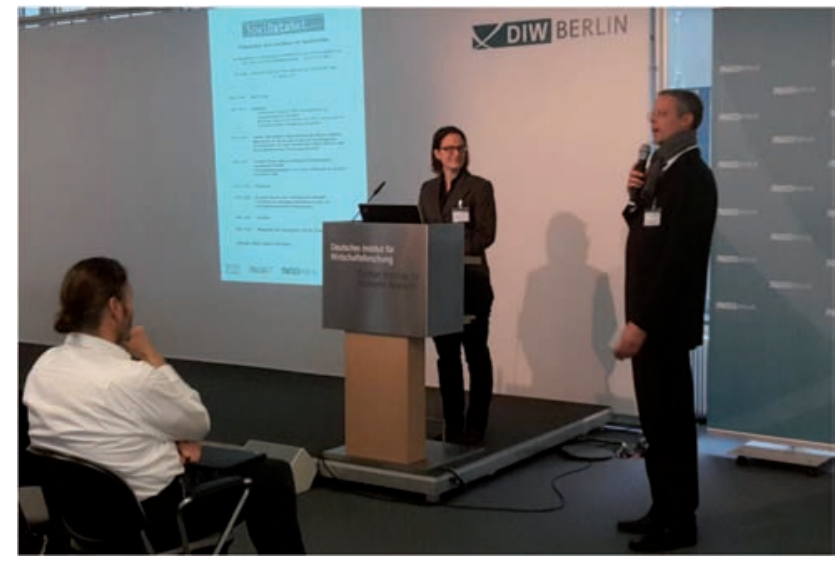

Abbildung 1: Moderator Mathis Fräßdorf (WZB Berlin) stellt die Projektleiterin Monika Linne (GESIS) vor (Foto: Claus-Peter Klas).

Im Anschluss präsentierte Monika Linne (GESIS - Leibniz Institut für Sozialwissenschaften, Projektleitung) die wesentlichen Ziele und Funktionen des „ForschungsdatenRepositoriums SowiDataNet für die Sozial- und Wirtschaftswissenschaften“.

Die Entwicklung dieser Infrastruktur orientiert sich am konkreten Bedarf institutioneller Anforderungen von Forschungseinrichtungen, die über keine eigene Forschungsdateninfrastruktur verfügen. Die Bedarfe dieser Zielgruppe wurden durch eine intensive Anforderungsanalyse in Form eines Workshops, durch Experteninterviews sowie Literaturanalysen erhoben. Dabei war sicherzustellen, dass der neue Service in den täglichen Arbeitsablauf des institutionellen Forschungsdatenmanagements flexibel eingebunden werden kann. Die Bereitstellung dieser digitalen Infrastruktur ermöglicht die Integration von dezentral produzierten Forschungsdaten und unterstützt das institutionelle Forschungsdatenmanagement $u$.a. durch drei spezielle Funktionalitäten.

1. Das „Dokumentationstool für Forschungsdaten“ erlaubt es, eigene Daten nach einheitlichen Regeln von den Forschenden institutsintern $\mathrm{zu}$ dokumentieren. Diese können nach einer anschließenden Qualitätsprüfung durch institutionelle Kuratorinnen und einer abschließenden Überprüfung durch GESIS mit SowiDataNet zitierbar veröffentlicht und erschlossen werden. Detaillierte Informationen können webbasiert zum Projekt, zur inhaltlichen Beschreibung der Daten und der Methodik der Untersuchung, sowie zu den Projektdateien erfasst werden. Neben Freitextfeldern werden ca. zwanzig kontrollierte Vokabulare, z. T. mit 
Autocomplete-Funktion, angeboten, die den Erfassungsaufwand verringern. Neben der modifizierten Anwendung des DDI Standards zur Datendokumentation werden die Metadatenstandards Dublin Core und DataCite zur besseren Auffindbarkeit genutzt. Im Zuge der Datenregistrierung werden die einzelnen Datenprojekte mit einem DOI versehen. Um den Datenzugang zu kontrollieren, können die Forschenden einer Einrichtung flexible Zugangsrechte $\mathrm{zu}$ den Forschungsdaten festlegen.

2. Die „institutsinterne Kurationsfunktion“ ist Teil eines zweistufigen Prozesses zur Qualitätssicherung der abgeschlossenen Projekt- und Datendokumentation. Dabei werden die erfassten Informationen und eingereichten Dateien mit Hilfe transparent definierter Qualitätskriterien geprüft, um die Sicherung und Nachnutzbarkeit der Daten mit der Infrastruktur SowiDataNet langfristig zu ermöglichen. Damit wird auch das Ziel verfolgt, Umfang und Qualität der Dokumentationstätigkeiten der Wissenschaftlerinnen im Forschungsdatenmanagement anzuerkennen und damit die Reputation der Forschenden und ihrer Institution $\mathrm{zu}$ fördern. Daten und Begleitdokumentationen werden in dem zweistufigen Prozess zuerst institutionell kuratiert und offene Fragen zur Einreichung mit den Forschenden der Institution besprochen. Die abgeschlossene institutionelle Prüfung der Einreichung wird dann zur Aufnahme in das GESIS-Datenarchiv an den GESIS-Kurator weitergeleitet, der die Kuratierungsangaben des Instituts sichtet. Ergeben sich auf dieser zweiten Stufe der Qualitätsprüfung noch Fragen, werden diese unmittelbar mit der Kuratorin der Institution besprochen. Das Verfahren erfolgt digital gestützt nach standardisierten Abläufe und Kriterien, die ausführliche in einem User-Guide dokumentiert sind. Mit Hilfe von System-Checklisten werden dabei drei Schritte durchlaufen. Im Zuge der technischen Prüfung werden die Lesbarkeit der Dateien, Lizenzund Urheberrechte sowie Datenschutzrechte überprüft. Die inhaltliche Prüfung dokumentiert den Dateneingang, die Art und Weise der Datenaufbereitung, die verwendeten Metadaten(-standards) sowie die bereitgestellte Begleitdokumentation. Im letzten Schritt erfolgt eine Zusammenfassung der Qualitätsprüfung. Nach Abschluss des Reviews werden die eingereichten Daten und Dokumentationen für mindestens fünfundzwanzig Jahre im GESIS Datenarchiv langfristig gesichert. Die Arbeit der Kuratorinnen wird durch intensive Betreuung mit persönlichem Support, Workshops und Vernetzung begleitet, um den Service mitzugestalten und weiterzuentwickeln.
3. Die Infrastrukturfunktion „Lokale Vitrine“ ermöglicht es, der jeweiligen Institution ihre mit SowiDataNet erfassten, gespeicherten und qualitätsgesicherten Forschungsdaten und Dokumentationen lokal in den Instituts-Webauftritt zu integrieren (Institutionelle Ansicht), um diese so zur Information und Nachnutzung anzubieten. Die derart integrierte Kollektion von Forschungsdaten eines Instituts wird im eigenen Corporate Design angezeigt und erhöht damit auch die Sichtbarkeit der zugrundeliegenden Forschungsprojekte nach außen. Gleichzeitig können Forschende über die Instituts-Website durch den eigenen Datenbestand browsen und ihn mit Hilfe der facettierten Suche jederzeit gezielt erschließen (Institutionelle Datenbibliothek). Die Motivation von Wissenschaftlern, Daten zu dokumentieren und zu veröffentlichen, wird damit unmittelbar auf Institutsebene gefördert, indem die Sichtbarkeit von Forschungstätigkeiten und publizierte Datenanalysen innerhalb und außerhalb des Instituts dauerhaft erhöht werden. Durch die Vernetzung von dezentral erzeugten Forschungsdaten sind die eigenen Angebote über die „Zentrale Recherche“ im SowiDataNet-Gesamtkatalog verfügbar.

Zum Geschäftsmodell von SowiDataNet stellte Linne folgende Eckpunkte vor. Die Nutzung soll während der Betaphase (pro Institut zehn Projekte, je 1 GB) und in ersten sechs Vertragsmonaten (pro Institut fünf Projekte, je $1 \mathrm{~GB}$ ) entgeltfrei sein. Für den dauerhaften Regelbetrieb ist ein Grundpreis pro Institut in Form einer jährlichen Kostenpauschale vorgesehen. Zur anteiligen Refinanzierung der variablen Kosten (Kostenentstehung durch Dateneinreichung) ist ein einmaliger Preis pro Projekt (max. 1 GB, Laufzeit mind. 25 Jahre) geplant. Konkrete Kostenangaben sollten bis Ende März 2017 vorliegen.

Drei Live-Demonstrationen untermauerten den weit fortgeschrittenen Entwicklungsstand von SowiDataNet. Monika Linne erläutere die Bereiche des Tools zur Datendokumentation, die Suchfunktionen und die Vergabe eines DOI. Patric Droß (WZB) führte dann durch den Workflow der Kurationsfunktionen und erläuterte, wie die Arbeitsabläufe und die Kommunikation zwischen GESIS, den institutionellen Kuratoren und den jeweils beteiligten Forschenden gestaltet ist. Timo Borst (ZWB) zeigte schließlich, wie die Projekte und produzierten Daten der Forschenden mit der lokalen Vitrine dargestellt werden, wie sie durch die facettierte Suche erschlossen und wie die Kollektionen der Institute in den Gesamtkatalog integriert sind. Neben technischen Aspekten des Systems erläuterte er, wie die Informationen der GND (Gemeinsame Normdatei) speziell für den Bedarf der Sozial- und Wirtschafts- 


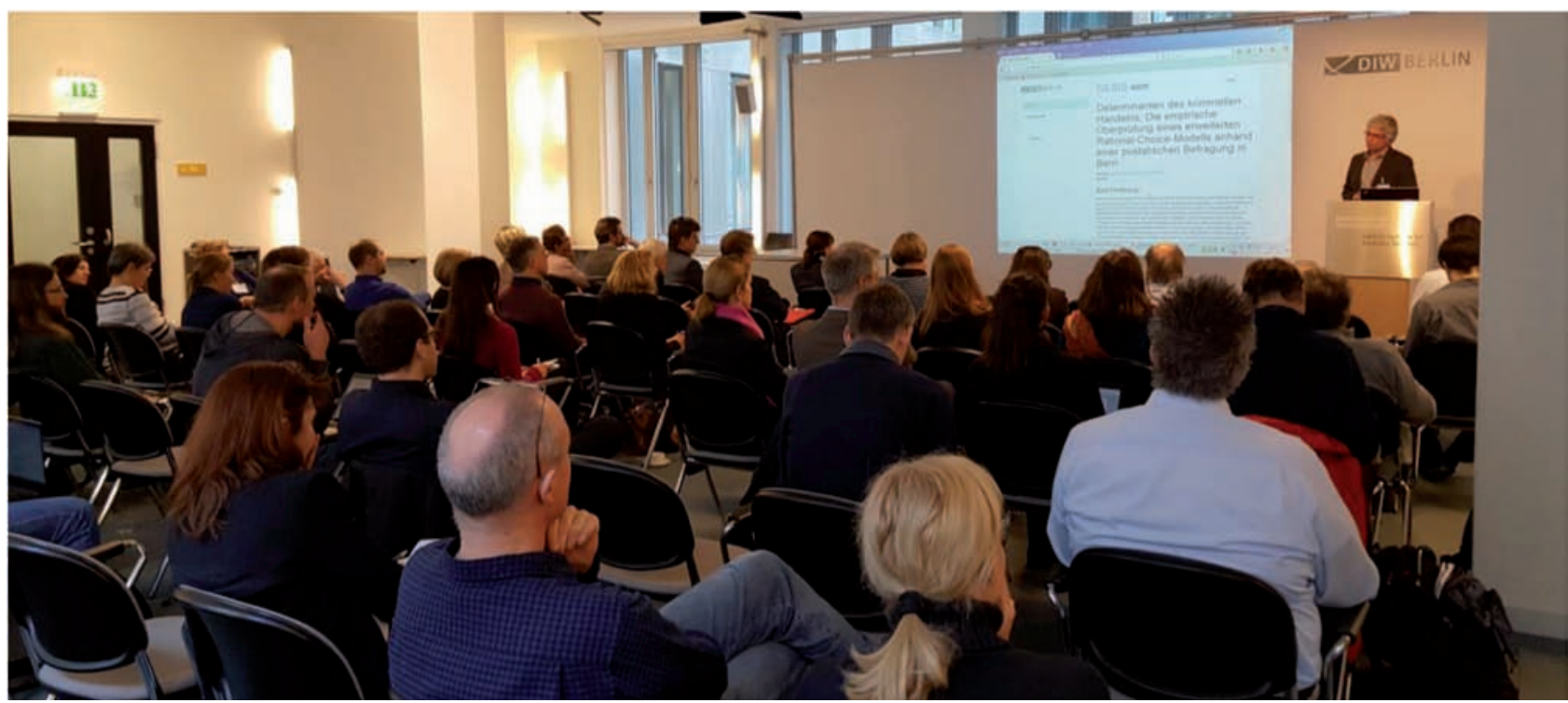

Abbildung 2: Live-Vorführung der Anwendung „Lokale Vitrine“ durch Timo Borst (ZBW) (Foto: Claus-Peter Klas).

wissenschaften gefiltert und verknüpft werden können, um sie gezielt in SowiDataNet zu nutzen.

Die Präsentationen und Live-Demo stießen auf ein lebhaftes Echo unten den Teilnehmenden, die viele Nachfragen zu einzelnen Funktionen und Arbeitsabläufen stellten. Insgesamt erscheint das Interesse an dieser digitalen Infrastruktur zur Unterstützung des institutionellen Forschungsdatenmanagements groß zu sein: 35 unterschiedliche Einrichtungen (44 Personen) haben sich auf der Veranstaltung für einen Testzugang angemeldet, um sich ein detailliertes Bild von SowiDataNet machen zu können. Die Testphase startet im April 2017. Im Mai 2017 beginnt die Beta-Phase des Produktivsystems, welches als Version 1.0, einschließlich Mehrsprachigkeit und Versionierung, online geht.

Deskriptoren: Tagung, Datendokumentation, Wissenschaft und Technik, Forschungsdaten

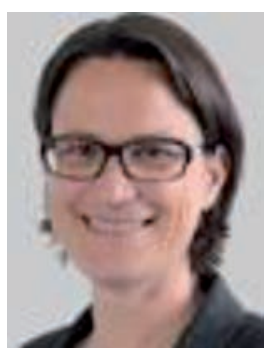

\section{Monika Linne}

GESIS Leibniz-Institut für

Sozialwissenschaften

Unter Sachsenhausen 6-8

50667 Köln

ORCID: 0000-0003-3526-6522

monika.linne@gesis.org

Monika Linne studierte Soziologie an der Universität Köln (Abschluss Magistra Artium). Danach arbeitete sie für die Bundeszentrale für gesundheitliche Aufklärung (BZgA) im Bereich von Gesundheitsinformationssystemen. Anschließend war sie als Projektmanagerin für
Medienanalysen bei der Unicepta Gesellschaft für Medienanalyse mbH tätig. Seit 2010 ist sie wissenschaftliche Mitarbeiterin bei GESIS im Datenarchiv für Sozialwissenschaften und leitet u. a. das Projekt SowiDataNet. Darüber hinaus promoviert sie zum Thema Intraorganisationale Soziale Netzwerkanalysen.

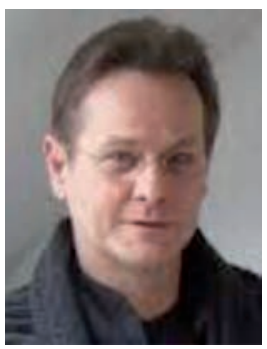
Uwe Jensen
GESIS Leibniz-Institut für
Sozialwissenschaften
Unter Sachsenhausen 6-8
50667 Köln
ORCID: 0000-0003-3579-6005
uwe.jensen@gesis.org

Uwe Jensen studierte Psychologie an der Universität Köln (Abschluss Diplom-Psychologe). Seit 1995 ist er wissenschaftlicher Mitarbeiter und befasst sich im GESIS Datenarchiv für Sozialwissenschaften mit Standards, Archivprozessen und Fragen des Forschungsdatenmanagements. Seine Expertise hat er in zahlreiche nationale und internationale Infrastrukturprojekte für die Sozialwissenschaften eingebracht; aktuell leitet er das AP3 Metadatenstrukturen des SowiDataNet Projekts. 